\title{
Une vision de la "fabrique de la parole" en cours de langue à travers des verbalisations d'enseignants
}

Genres et normes interactionnels

Francine Cicurel

\section{OpenEdition}

\section{Journals}

Édition électronique

URL : http://journals.openedition.org/rdlc/693

DOI : $10.4000 /$ rdlc.693

ISSN : 1958-5772

Éditeur

ACEDLE

\section{Référence électronique}

Francine Cicurel, «Une vision de la "fabrique de la parole" en cours de langue à travers des verbalisations d'enseignants », Recherches en didactique des langues et des cultures [En ligne], 12-2 | 2015, mis en ligne le 20 novembre 2015, consulté le 01 mai 2019. URL : http:// journals.openedition.org/rdlc/693; DOI : 10.4000/rdlc.693

Ce document a été généré automatiquement le 1 mai 2019.

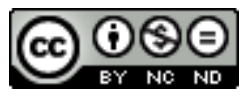

Recherches en didactique des langues et des cultures is licensed under a Creative Commons AttributionNonCommercial-NoDerivatives 4.0 International License 


\title{
Une vision de la "fabrique de la parole" en cours de langue à travers des verbalisations d'enseignants
}

Genres et normes interactionnels

\author{
Francine Cicurel
}

\section{Introduction}

1 S'appuyer sur les paroles d'enseignants confrontés à leur action en classe permet d'obtenir le jaillissement de commentaires sur l'action passée. Que peut-on attendre comme type de savoirs émanant de ces dires d'enseignants de langue, qu'est-ce que l'enseignant découvre sur lui, sur sa manière de faire cours, sur ses interlocuteursapprenants quand il devient observateur de lui-même en train d'agir? L'analyse de ces autocommentaires qu'un certain nombre d'enseignants ont produits au moment du visionnement de leur propre cours de langue et que nous avons eu l'occasion de recueillir ${ }^{1}$ nous a permis de voir que, bien qu'il s'agisse de l'enseignement d'une langue, l'objet principal des discours de commentaires d'enseignants ne se construit pas principalement autour de faits de langue ou de contenus langagiers à transmettre. Qu'est-ce à dire ? L'objet disciplinaire est pourtant organisé autour de données langagières à transmettre et qui, la plupart du temps, font problème ! La langue serait-elle absente des préoccupations des enseignants dont l'effort porte pourtant essentiellement sur l'acquisition de compétences langagières? Tout professeur de langue cherche à un moment ou un autre à "faire parler" les élèves, il est tenu de jouer son rôle de transmetteur et d'évaluateur. L'interlangue des apprenants donne lieu à évaluation, reprises, reformulations. Or, on s'aperçoit que l'objet des autocommentaires, plutôt que de porter sur le code, ou sur les difficultés de la langue tend à évoquer les pratiques langagières des publics d'apprenants et la manière dont l'enseignant lui-même est amené à s'impliquer dans ces pratiques. À cette occasion, alors que l'enseignant signale en quelque sorte des "incidents", émerge 
l'existence de normes interactionnelles dont on fait l'hypothèse qu'elles font partie intégrante de la fabrique de l'action en classe de langue.

Nous nous appuierons sur des verbalisations d'enseignants sollicités par un enquêteur à réagir de façon spontanée à leur propre cours filmé. A la lumière d'un corpus constitué par un corpus de verbalisations d'enseignantes ${ }^{2}$ de cours de FLE, on tentera d'ouvrir une fenêtre sur la manière dont se fabrique la parole en classe.

\section{Trois remarques en guise de préambule}

\section{Remarque liminaire 1 : connaître/ reconnaître les genres conversationnels}

3 L'interaction didactique a ses traits spécifiques et est elle-même un genre discursif ; elle constitue le cadre de l'action mais, en son sein, apparaissent des sous-genres de discours, des manières de formater les activités langagières. Ce qui retient ici notre attention est la question des formats discursifs tels que les enseignants les énoncent. Dans un chapitre intitulé "Le savoir socioculturel dans l'inférence conversationnelle", Gumperz (1989: 70) donne la définition suivante d'une activité langagière : "un ensemble de relations sociales qui s'effectuent selon des schémas articulés à un but communicationnel donné".

4 Par exemple, parler du temps, discuter politique, bavarder autour d'un café, sont des activités de langage que des acteurs sociaux connaissent et reconnaissent. Ces derniers vont, par conséquent, avoir des attentes quant aux règles de l'interaction à laquelle ils vont participer, au format, aux enjeux. Ainsi, dans une discussion entre amis, le sujet est libre alors que dans une conférence, l'auditoire attend qu'un sujet $\mathrm{x}$ soit traité par le conférencier.

Dans l'apprentissage/enseignement d'une langue, la question se pose de savoir ce que l'on considère comme étant "la langue" que l'on veut transmettre : s'agit-il de contenus langagiers, organisés selon des progressions pédagogiques, de discours sur la langue, de l'usage de cette langue par les participants, par les natifs, du déploiement d'activités didactiques appropriées ? Sans doute peut-on répondre par l'affirmative à ces questions, mais qu'en pensent les professeurs eux-mêmes? Qu'ont-ils à dire sur la langue, celle qui est à transmettre et à apprendre ? Curieusement, alors que dans l'interaction elle-même la dimension métalinguistique est au premier plan (voir Cicurel, 1985), lorsque les enseignants interrogés commentent leur cours, la focalisation sur la langue, dans son aspect descriptif, n'apparaît que rarement : pas ou peu de commentaires sur les règles, le lexique, la prononciation. La langue en tant que telle serait-elle absente de la conscience réflexive de l'enseignant? De quelle manière le "souci de la langue" va-t-il alors apparaître, que va-t-on apprendre de la manière dont l'enseignant s'arrange avec ce qu'il doit transmettre? En regardant de plus près les verbalisations, on va cependant découvrir que la langue est loin d'être absente, mais qu'elle se présente comme une activité de langage, comme un mouvement, qui fait lien et va d'un locuteur à un autre selon un certain format. 


\section{Remarque liminaire 2 : l'auto-confrontation comme moyen d'investigation de l'action}

6 Il faut donner ici quelques informations quant à la méthodologie de recueil de données qui sous-tend notre démarche. En effet comment en arrive-t-on à déterminer quelquesuns de ces traits propres à l'agir professoral? C'est au cours d'entretiens d'autoconfrontation ${ }^{3}$, durant lesquels on demande à des professeurs de commenter le film de l'un de leurs cours, que l'on suscite une mise en mots de l'action. Ces entretiens d'autoconfrontation donnent accès aux perceptions que le professeur a de sa propre action. La méthode que l'on appelle auto-confrontation (ou stimulated recall, cf. Gass \& Mackey, 2000) a pour particularité de réveiller le souvenir évanescent. Au moment de son action en classe, l'enseignant trop pris par cette action, ne peut avoir une posture réflexive mais, a posteriori et dans certaines conditions, il va retrouver quelques-uns des motifs, des émotions, des intentions qui étaient les siens. Le postulat de la conscience préréflexive de Theureau (2010) ${ }^{4}$ est à la base de notre réflexion. Il pose qu'un individu peut commenter, après coup, des éléments qui ont présidé à la réalisation de son action. Placé devant une trace de son action - l'image filmée - il convertit cette action en discours et en propose des significations. Pour autant, l'action ne se laisse pas obturer par le discours produit, mais il devient possible de dégager des schèmes d'action à partir de l'image filmée commentée par le sujet-professeur.

7 Les recherches entreprises par le groupe Idap du Diltec s'appuie sur des verbalisations de professeurs de langue placés devant le visionnement de leur cours. Autrement dit, il est demandé à des praticiens d'adopter une attitude réflexive en revenant sur ce qu'ils ont fait dans une classe, une reconstruction de l'expérience en quelque sorte. Ces "paroles sur l'action" permettent de trouver des traces de la manière dont un enseignant se représente la transmission d'une langue à enseigner, les obstacles, les stratégies et tactiques à mettre en place pour atteindre les buts fixés. À travers ces verbalisations, une porte s'ouvre sur la "pensée enseignante" (Aguilar \& Cicurel, 2014). Verbalisation est un terme que l'on utilise pour parler de la mise en discours d'une action ou d'une activité idéelle par des mots 5 . Par ce dispositif, on encourage un acteur social à revenir sur une action accomplie et à en faire un commentaire.

Quels sont les premiers constats que l'on peut faire devant cette parole commentative de l'action? On peut dire qu'elle est foisonnante, intense et parfois déconcertante. Mais si, à première audition ou lecture, elle parait décousue voire disloquée, après une analyse plus minutieuse, des lignes de force plus stables peuvent être dégagées. En effet, l'action du professeur en classe est multimodale, elle va simultanément dans plusieurs directions, usant de moyens divers - parole, geste, écriture, usage de supports ${ }^{6}$. Par conséquent, devant le filmage de son cours, le professeur qui verbalise son action a toujours plusieurs choses à dire en même temps. Il commente l'attitude des interlocuteurs, ses propres intentions, les gestes, la réception de son discours, il donne des informations sur sa pratique, il s'étonne, il commente les interventions apprenantes, il rationalise, etc.

\section{Remarque liminaire 3 : de l'action à la fabrique de l'action}

Avoir accès à l'agir professoral dépasse le cadre de l'observation de la classe puisqu'il est fait appel aux représentations que le sujet agissant propose de l'action accomplie. On 
considère dans cette optique qu'un retour sur l'action est possible. Or, selon la philosophe Arendt, ce qui caractérise l'action c'est qu'elle a pour propriété d'être effacée aussitôt produite.

10 Arendt $^{7}$, abordant les divers modes de l'activité humaine, établit une distinction entre fabrication et action. La fabrication est une activité qui vise à produire des objets au sein desquels on peut distinguer ceux destinés à la consommation courante et les œuvres d'art (susceptibles de prétendre à une certaine immortalité car ils résistent à l'usage infligé aux objets de consommation courante). Les objets durent généralement plus longtemps que l'activité qui les a fabriqués, la durée de fabrication des objets est connue. En revanche les actions des hommes sont évanescentes. Elles disparaissent sitôt accomplies. À moins qu'elles ne soient consignées par l'histoire ou la littérature. Une autre caractéristique de l'action est son infinitude. Une action est rarement isolée, elle en engendre une autre, qui à son tour exerce une influence sur l'un ou l'autre des acteurs. De telle sorte qu'il peut paraître impossible d'assigner à l'action un début et une fin.

11 Les verbalisations d'enseignants se produisent devant une action accomplie en classe, destinée à tomber, du moins partiellement, dans l'oubli. Le fait de pérenniser un cours, déterminé dans le temps, retenu ou revenant en mémoire grâce au filmage, change le statut de l'action. Sans aller jusqu'à dire qu'on tend à fabriquer une action-cours, il faut bien constater que sans le filmage, sans la transcription, les actions accomplies pendant le cours et les intentions qui les accompagnent seraient oubliées. Le professeur placé dans ce dispositif a l'occasion (qui lui est rarement donnée) de mettre en mots son action, de revenir sur sa pensée (les intentions, les motifs, les jugements, les conceptions, ce qui préside aux décisions, etc.). Il contribue ainsi à la fabrication d'une entité qui peut paraître en partie artificielle (dans le sens où ce n'est pas ainsi que cela se passe habituellement) mais qui fixe dans le temps l'action et la pérennise. C'est à partir de ce matériau discursif, pour la constitution duquel praticien et chercheur coopèrent, que peut se déployer une ouverture sur l'action en classe.

\section{Quelles représentations du cadre-classe ; normes interactionnelles d'une parole en cadre institutionnel}

12 Apprendre une langue en situation institutionnelle possède des traits spécifiques qui différencient cette situation de l'appropriation langagière en milieu naturel. Enseigner ou apprendre une langue peut être représenté par le schéma suivant :

\begin{tabular}{|l|l|l|}
\hline $\begin{array}{l}\text { L1(locuteur } \\
\text { compétent) }\end{array}$ & doit/veut transmettre & $\begin{array}{l}\text { à Ls (locuteurs } \\
\text { moins compétents) }\end{array}$ \\
\hline & des connaissances, un savoir-faire/savoir-dire & \\
\hline selon des méthodes et moyens X & pour accélérer les processus acquisitionnels \\
\hline $\begin{array}{l}\text { par la médiation d'objets (souvent scripturaux : textes, livres, outils, inscriptions au tableau, } \\
\text { technologies informatiques) }\end{array}$ & \\
\hline & dans un lieu et un temps donnés
\end{tabular}


- influence du contexte, géographique, institutionnel, de ce qui est en-dehors de la classe (statut de la langue apprise, possibilités d'entendre la langue, de la parler, etc.)

- contrat didactique : accepter de part et d'autre de se plier à certaines activités langagières dans un but d'appropriation de savoirs

13 Comment l'enseignant se situe-t-il par rapport à ce qu'il convient d'appeler un cadre? Le caractère prédéterminé de l'interaction (buts d'apprentissage, programme et contraintes institutionnelles) n'exclut pas pour chacun des participants la possibilité de prendre la parole, ce qui conduit l'interaction à prendre un tour qui n'est jamais totalement prévisible. Se profile ici pour les enseignants de langue une double contrainte : enseigner une langue en contexte institutionnel demande à la fois à ce qu'il y ait maintien de la didacticité de l'interaction, mais aussi qu'il puisse y avoir une prise de parole individualisée et spontanée, toujours susceptible de conforter l'ordre interactionnel, de le déranger ou le faire dévier. On n'est pas loin d'une communication paradoxale : il faut permettre aux apprenants de "parler naturellement" mais ceci dans le respect de règles liées à la situation didactique qui sont différentes des règles de communication en vigueur à l'extérieur de la classe (voir les exemples donnés par Cicurel 2011 dans le chapitre "La classe et son extérieur").

Rappelons qu'exercer le métier d'enseignant renvoie à un "genre professionnel", c'est-àdire à une "culture d'enseignement" que partagent des professeurs d'une communauté donnée, autour d'une matière à enseigner. La notion de "genre professionnel" a été développée dans le domaine de l'analyse du travail par Clot \& Faïta (2000) qui le considèrent comme étant constitué par un stock d'actes, de routines, de conceptualisations "prêts à servir". L'action est possible parce qu'il existe dans le contexte des professionnels un savoir langagier, social et professionnel partagé qui permet à cette action de se réaliser au sein d'un genre professionnel. Ce sont les "formes communes de la vie professionnelle" qui font que l'on sait, dans un contexte donné, comment s'adresser à l'autre, comment mettre en place une activité, la terminer, organiser une réunion, savoir ce que l'on attend de vous. Cela renvoie à la connaissance qu'un sujet a des manières de parler dans une situation donnée (Gumperz, 1989). Cela signifie donc que le métier exige de se conformer à certaines normes qui sont en quelque sorte la marque de fabrique de la profession.

15 Tournons-nous à présent vers quelques extraits de verbalisations ${ }^{8}$ et tentons de voir si la référence au cadre professionnel apparaît et de quelle manière.

\section{La représentation du format-cours : un cadre de référence pour les enseignants?}

Tout enseignant, avons-nous dit, appartient à un genre professionnel marqué par des gestes et des actions typiques9. L'action est menée selon les schèmes d'un idéal incorporé. Dans cet idéal se trouve représentés le comportement que l'on vise mais aussi la représentation de ce qu'est un cours. C'est lorsque des incidents surgissent qu'un individu amené à réaliser une tâche prend conscience des conditions nécessaires pour exécuter la tâche. Dans l'extrait ci-après, nous voyons que l'enseignante s'exprime à propos de l'une des apprenantes qui cherche à capter son attention, ce qui, selon elle, risque de la détourner de ce qu'elle estime être ses obligations professionnelles ${ }^{10}$. 


\section{Corpus Corny}

qu'on me parle sur le plan personnel ça arrive souvent qu'on me capte même parfois on m'attrape le bras j'ai j'ai beaucoup de mal je peux pas m'extraire de ce contact-là ++ pendant ce temps-là peut-être que les autres peuvent discuter se disent bon c'est une relation entre elles donc nous on discute ++ donc quand ça dure quelques secondes tout c'est pas très grave mais quand je vois qu'la chose s'installe après c'est TRES difficile si tu veux de revenir justement sur le sujet et c'est pas tout à fait un salon de thé c'est quand même un cours ++ et je pense que la difficulté c'est toujours de tenir une tension quand même + euh bon vous avez le droit de parler vous avez le droit de vous exprimer y'a du français du cambodgien euh plutôt français de préférence quand même ++ mais quand même y'a un cours et on on on parle d'une matière en commun quoi c'est pour tout le monde voilà + et là c'est l'exemple typique où: parce que là je voyais à à la à l'écran elle me regarde encore jusqu'au BOUT si tu veux elle me lâche PAS au niveau de la communication + donc et moi et après je reformule pour les autres

Que pouvons-nous tirer comme connaissance de l'idée que se fait Valérie à propos de la manière dont il faut tenir une classe? Que considère-t-elle comme "grave" ou transgressif ? Pourquoi l'aparté avec une apprenante pourrait-il être dangereux ? Devant cet "incident conversationnel", Valérie est amenée à énoncer ce qu'elle considère comme étant les règles didactiques à respecter : pour qu'il y ait cours, il faut qu'il y ait travail en commun: "mais quand même y'a un cours et on parle d'une matière en commun quoi c'est pour tout le monde voilà".

Elle mentionne également la durée de cette interaction privée, en aparté, car selon elle la durée excessive compromettrait la possibilité de se concentrer ultérieurement sur le thème du cours. Il n'est pas facile pour l'enseignante de parvenir à formuler cette "règle conversationnelle" car son commentaire montre par ailleurs qu'elle hésite constamment entre le respect du droit à parler des apprenantes et l'obligation qu'elles se conforment à la règle générale. Les mots qu'elle utilise nous permettent de comprendre la définition qu'elle se donne d'un cours, à savoir qu'il y ait travail en commun sur un sujet donné par un enseignant et selon des modalités qui ne sont pas celles des discussions libres en petits groupes.

9 L'enseignante Valérie exprime là un dilemme que l'on retrouve souvent dans les verbalisations d'enseignants de langue. Faut-il accepter une "interaction privée" avec un apprenant, faut-il porter attention à l'un des interactants, au risque de se désintéresser des autres? C'est un choix à faire, l'un des termes de l'alternative va dans le sens de la faveur que l'on donne à la communication et de l'importance à accorder à la personne ; mais il entre en conflit avec l'autre terme du dilemme: que font les autres pendant ce temps ? Vont-ils être livrés à eux-mêmes ? Vont-ils bavarder? Écouter d'une oreille ? Et peut-être alors perdre leur temps! C'est bien à propos du genre conversationnel classe que s'est exprimée ici l'enseignante. Genre qui a des règles communicatives sans doute mais qui, dans le concret de la situation, ne sont pas simples à mettre en œuvre.

On observe que l'enseignante s'interroge : "où est-on? Dans un salon de thé ou dans un cours", ce qui l'amène à repréciser le format-cours: les participants sont là pour apprendre, son rôle à elle est de permettre d'apprendre. Il y a un programme, des objectifs, donc des normes qui ne sont pas compatibles avec la spontanéité de la conversation ordinaire. 
21 Le genre professionnel implique que l'on respecte le format du cours et les obligations liées à l'interaction didactique. Il faut parfois savoir renoncer à certaines actions pour sauver l'interaction. En tant que personne, ce jeune professeur aimerait entrer en contact personnel avec l'étudiante désireuse de lui parler mais la conscience d'être un "professeur" et d'avoir à "faire un cours" vient contrarier cette envie. Elle reprend alors la gestion des activités à finalité didactique, et par son commentaire livre à l'analyste ce que doit être, selon elle, un cours de langue :

- Il doit comporter un objectif langagier,

- Cet objectif doit être utile à tout le groupe,

- Le cours ne peut être seulement une conversation mondaine.

\section{Les normes interactionnelles liées au genre « appel »}

Observons une petite séquence intervenant alors que Valérie commente le début de son cours. Il y est question de l'activité "faire l'appel".

\section{Corpus Corny}

Le classeur d'appel je le fais circuler à la fin en général je l'oublie parce que euh leur présence en cours en extrêmement contrôlée par Tang et est sanctionnée s'il y a des absences + moi j'ai pas la culture de l'appel point je l'oublie facilement donc c'est même elles qui me rattrapent eh + euh on n'a pas signé voilà

Les locuteurs d'une communauté donnée savent, en général, comment se comporter verbalement et proxémiquement, selon ce qu'on appelle le "savoir pratique" de locuteurs. L'appel peut être considéré comme un genre discursif, à tout le moins une activité discursive ritualisée, qui a cours dans certains lieux comme la caserne ou l'école. Dans un cadre scolaire, tout participant peut s'attendre à être "recensé".

Dans le cas évoqué plus haut, ce sont les apprenantes qui réclament la mise en place de ce genre conversationnel, qui fonctionne un peu comme un marqueur d'entrée dans une interaction-cours et qui a une fonction sociale. L'"appel" est un genre conversationnel en lien avec le fonctionnement d'une institution qui "surveille". L'enseignante se définit par rapport à cette norme et prend des distances envers une pratique dont elle estime qu'elle ne la représente pas comme personne. "Moi j'ai pas la culture de l'appel" déclare-t-elle. Elle donne sa position, en usant d'une formulation intéressante comme si "culture de l'appel" renvoyait à des pratiques bien identifiées dans une communauté donnée. Ce faisant, elle livre un aspect du style d'enseignement qui est le sien. Elle indique ici qu'entre ce qui est demandé par l'institution ou l'organisme employeur et qui représente une pratique verbale canonique d'un cours et elle-même, en tant que personne, existe un hiatus. Sous l'enseignante se dévoile une personne ${ }^{11}$ prenant des distances par rapport à des habitudes de parole routinisées dans un contexte social.

\section{Conversation didactique vs conversation ordinaire ou le dilemme du "qui suis-je ?"}

L'extrait suivant pose la question des conditions d'émergence d'une conversation qui se déroulerait en classe comme à l'extérieur, en incluant des rôles symétriques et sans 
mobile connu à l'avance. Il s'agit toujours d'une séquence de verbalisation de l'enseignante Valérie, alors qu'elle commente le début du cours.

En général les dix premières minutes euh ::: c'est une conversation un p'tit peu: sans objectif particulier euh: soit on résume un p'tit peu c'qui s'est passé le week end soit euh mais en général c'est une conversation je prends un peu un prétexte de la fenêtre ou quoi que ce soit pour essayer de de reprendre contact en fait c'est une façon de reprendre contact après une semaine euh voilà

Une classe de langue se caractérise par la mise en place d'activités didactiques; en effet, enseigner la langue, c'est travailler à partir de "parties de langue", formatées ou incrustées dans des exercices et activités pédagogiques orientées selon des objectifs d'apprentissage. La mention que fait l'enseignante Valérie : "c'est une conversation un petit peu sans objectif" mérite qu'on s'y arrête. L'activité de langage "conversation" est connue d'individus qui, vivant en société, savent qu'il s'agit d'échanges de propos libres entre des interactants généralement dans une place symétrique, les thèmes abordés n'étant pas imposés de l'extérieur. Que veut dire alors, dans la bouche de l'enseignante, la qualification "sans objectif" alors qu'une conversation ordinaire n'est pas censée avoir un objectif? Il ne peut s'agir que d'une référence au fait que, dans une classe de langue, on attend toujours qu'il y ait un objectif didactique puisque précisément on est là pour apprendre.

On constate à travers l'énoncé "je prends un prétexte de la fenêtre (qui doit être ouverte)" que la conversation en classe ne va pas de soi. Il n'est pas impossible qu'on ait là comme les termes d'une contradiction: vient-on pour converser ou pour apprendre (Bigot, 1996), qui va être le leitmotiv de ce que dira ce professeur tout au long de sa verbalisation ${ }^{12}$. Le contrat de la classe, comme on l'a mentionné plus haut, est de permettre qu'il y ait de l'apprentissage. Une conversation "sans objectif particulier" révèle le paradoxe : peut-on parler librement alors qu'on est formaté par les règles d'un cours?

presentés portaient sur des pratiques conversationnelles ritualisées : l'appel, la conversation, l'entretien privé, le format du cours. Il arrive que, selon les genres conversationnels, les places dans l'interaction bougent au risque de faire vaciller le rôle-professeur. L'interaction didactique se caractérise par un clivage entre deux places dans l'interaction non rapportables l'une à l'autre: celle de celui qui sait et qui doit enseigner et celle de ceux qui sont là pour apprendre. On constate à travers les verbalisations que cette place n'est pas statique ou immuable, et qu'elle donne lieu à réflexion ou doute de la part de l'enseignante. L'enseignant se laisserait-il parfois entraîner dans une interaction où il prendrait un rôle conversationnel symétrique de celui de ses apprenants, risquant par là de modifier le cadre-classe?

Les enseignants ont des positions assez personnelles sur la gestion des genres conversationnels et prêter attention à leurs paroles montre comment ils se situent au sein d'un genre professionnel. 


\section{Bavardage et silence : une perception des interventions apprenantes}

Comme on le sait, et cela a été documenté, l'enseignant est celui qui gère l'interaction. La fréquence du schéma ternaire sollicitation/ réponse/ réaction (Sinclair \& Coulthard, 1975) montre qu'il est le médiateur de la parole en classe. C'est lui qui la provoque en lançant questions et consignes, c'est lui qui indique qui doit prendre la parole, lui qui canalise cette parole et qui l'évalue. Dans ce sens, il est bien un "fabricant de parole". Sans lui, l'interaction ne se produirait pas.

Reprenons la question, souvent exprimée par les enseignants, du désir que leurs élèves prennent la parole. Mais cette parole apprenante peut-elle être prise dans n'importe quelles conditions?

\section{Le "bavardage" : un genre conversationnel propre à la classe?}

Dans les verbalisations d'enseignants émerge comme une image du cours tel qu'il devrait se dérouler. Si le souci de faire respecter le format du cours se lit fréquemment, celui de faire en sorte que les apprenants parlent est lui aussi récurrent. Cette autre enseignante, Gaya, qui s'adresse à des étudiants chinois étudiant le français en France, s'inquiète de la non parole, du respect excessif pour le professeur qui, selon elle, paralyse les étudiants chinois. Voyons un extrait qui traite du "bavardage". Est-il admis ? Pas vraiment, on va le voir.

\section{Corpus Gaya}

bon y a des moment où ça m'dérange + euh : le bavardage quand on bavarde d'autre chose je déteste ça + donc je le fais remarquer de temps en temps parce que ça arrive avec les mobile phones $\uparrow+$ avec etc. les : ok + mais le bavardage il est le produit d'un échange qui qui qui est lié au COURS : ça je trouve ça très bien + donc là je vais JAMAIS faire de la discipline à ce moment-là + parce que ce serait une casse XXX la couper d'une espèce de : de de comment dire de dynamique qui est en train de se mettre en place par rapport au cours $\uparrow+$ par rapport au vocabulaire $\uparrow+$ par rapport à ce dont on a parlé + donc c'est très important de laisser quelque chose qui a l'impression d'être un peu chaotique + arriver + bien sûr je surveille + je vais pas laisser : + mais je vais pas couper $\uparrow+$ quand il y a une dynamique comme ça + je veux absolument la laisser parce que ça veut dire que vraiment les étudiants sont:: impliqués dans le processus

L'activité langagière "bavardage/ papotage" ne répond pas à la définition du cours que se fait cette enseignante. Partons de ce qui peut être considéré comme une norme partagée par la communauté professionnelle : un cours est une activité orientée vers un but qui permet l'accroissement d'un savoir. Est-ce qu'un bavardage permet cet accroissement ? En tout cas, ce n'est pas la représentation commune que l'on peut avoir d'une interaction à but didactique. Dans toute culture, selon Gumperz, les usagers savent faire la différence entre les activités langagières, et donc faire la distinction entre "bavarder" et "faire cours". $\mathrm{Au}$ début d'une interaction sociale, les participants règlent leur façon de parler et il y a établissement de ce que Gumperz appelle un "rythme conversationnel". Il faut donc tomber d'accord sur l'activité en cours. Ce que l'enseignante souhaite préserver c'est la liberté 
qui caractérise une conversation informelle, mais à condition que la parole ainsi prise soit liée aux contenus du cours. On y découvre ce que ressent ce professeur devant le bavardage : "Ca me dérange... Je déteste". Émergent des représentations quant au métier du professeur où les affects ne sont pas absents, pas plus que l'inquiétude quant à la gestion de la parole des apprenants : comment occuper sa place, jusqu'où peut-on laisser aller la liberté des étudiants?

\section{Le poids du silence}

"C'est toujours le silence qui met le monde sous un autre jour. Le silence de la neige en hiver. Le silence des jours fériés. Et puis là, le silence qui n'est pas le nôtre car nous n'en faisons pas partie, nous sommes extérieurs à ce silence", écrit l'auteur norvégien Jacobsen ${ }^{13}$.

C'est peut-être cette étrangeté du silence et son effet d'exclusion que ressent l'enseignante s'exprimant dans le passage qui suit. Que faire devant un public qui se tait, devant la non réponse ? Et arrive-t-il que l'enseignant, "maître de la parole", soit amené lui aussi à se taire? C'est ce que nous allons découvrir dans la verbalisation qui suit, dans laquelle l'enseignante Valérie propose une "explication" de son propre silence.

\section{Corpus Corny}

\begin{tabular}{|c|c|}
\hline Verbalisation du silence & Notre commentaire \\
\hline $\begin{array}{l}\text { alors t'as vu j'ai gardé un silence là je fais un gros effort et je vois } \\
\text { que je n'ai pas de réponse ++ }\end{array}$ & $\begin{array}{l}\text { Énonciation du } \\
\text { problème: parvenir à } \\
\text { rester silencieux devant } \\
\text { la non réponse. }\end{array}$ \\
\hline $\begin{array}{l}\text { parce que très souvent dans la culture je pense que c'est dans la } \\
\text { culture asiatique mais je mets ça avec énormément de guillemets je } \\
\text { ne sais pas + elles ont très peur de se tromper + y'a un rapport à } \\
\text { l'erreur + qui est + euh c'est pas sympa c'est pas + oh je lance une } \\
\text { réponse j'me suis trompée c'est pas grave je passe à une autre + donc si } \\
\text { tu veux y'a un enjeu c'est comme si y'avait un enjeu + du coup euh: + } \\
\text { euh un peu trop: + pesant + voilà qui fait que personne ne se lance + et } \\
\text { comme je m'identifie à elles j'me dis bon très souvent ça s'passe } \\
\text { comme ça }\end{array}$ & $\begin{array}{l}\text { Tentatives d'explication } \\
d u \quad \text { silence: culture } \\
\text { asiatique, rapport à } \\
\text { l'erreur, }\end{array}$ \\
\hline $\begin{array}{l}\text { j'me jette à l'eau la première comme si (rires) j'étais + élève et même } \\
\text { pas hein (rires) + donc je me mets un peu à un statut qu'est même } \\
\text { pas je quitte le statut du prof + j'leur montre soi-disant l'exemple } \\
\text { en disant bon allez + voilà moi j'me jette à l'eau alors voilà comment } \\
\text { j'ferais + parce que là j'vais faire ça euh::: je cherche en fait je cherche } \\
\text { pas hein + donc je j'vais mimer une scène où moi qui s'rais étrangère je } \\
\text { chercherais quelque chose dans ma tête que je ne comprends pas voilà } \\
\text { + et donc là je change mais ça c'est assez }\end{array}$ & $\begin{array}{l}\text { Séquence fictionnelle: } P \\
\text { joue à être élève. } \\
\text { Changement de place. }\end{array}$ \\
\hline
\end{tabular}




\begin{tabular}{|l|l|}
\hline $\begin{array}{l}\text { j'lai fait plusieurs j'l'ai fait souvent je + bon mais je pense que c'est } \\
\text { une stratégie de de + c'est une sorte d'exemple + d'exemple: + } \\
\mathbf{j} \text { 'donne l'exemple + c'est une forme d'exempli+fication c'est ça ? } \\
\text { voilà ++ donc euh voilà c'que j'voulais }\end{array}$ & $\begin{array}{l}\text { Stratégie non } \\
\text { généralement dans la } \\
\text { situation de séponse : s'identifier aux } \\
\text { apprenantes et montrer } \\
\text { l'exemple } \\
\text { De la pratique de l'action } \\
\text { aux savoirs d'action : elle } \\
\text { donne un nom à sa } \\
\text { stratégie: } \\
\text { exemplification }\end{array}$ \\
\hline $\begin{array}{l}\text { dire (rires) + mais là } \mathbf{j} \text { 'fais un effort et le silence là du coup ça } \\
\text { commence à m'angoisser (rires) }\end{array}$ & $\begin{array}{l}\text { Retour à la situation, } \\
\text { c'est-à-dire le silence. } \\
\text { Expression de l'émotion : } \\
\text { angoisse }\end{array}$ \\
\hline
\end{tabular}

L'enseignante commente le fait que, contrairement à ce qui se passe en général, elle s'efforce de garder le silence afin de donner le temps de la réflexion à son public. Mais elle se fait violence (je fais un gros effort) : prenant une attitude qui ne lui est pas naturelle, elle s'efforce de maintenir ce temps de latence, nécessaire, pense-t-elle, à l'élaboration des connaissances des apprenantes. Elle se lance dans la description de ce qu'elle fait $\grave{a}$ l'ordinaire quand elle ne se tait pas. On découvre la complexité de ce qui se passe mentalement en elle et combien le silence lui semble éprouvant.

Le visionnement de cette séquence - où d'évidence les apprenantes ne sont pas disposées à parler et où l'enseignante, elle aussi, garde le silence malgré la difficulté qu'il y a à le faire - pousse Valérie à approfondir les raisons pour lesquelles existent en classe des risques à laisser s'installer le silence : les apprenantes préfèrent ne pas prendre la parole de peur de se tromper. Il est fait référence à leur culture éducative où l'erreur est lourde de conséquences. L'enseignante va plus loin que le seul commentaire de ce qui se passe dans son cours car, en évoquant ce qu'elle fait d'habitude, à savoir ne pas laisser le silence s'installer, donner l'exemple en montrant comment on peut répondre, elle va exprimer ce que cette séquence lui apporte comme clé sur son propre comportement face au silence qui se prolonge. Ainsi la classe, lieu de parole, lieu pour faire produire de la parole, s'il se transforme en lieu de non parole, ne serait-ce que pour quelques instants, semble alors contenir l'indice d'un échec, celui d'une non-communication.

\section{Action et identité langagière}

L'action d'enseigner n'est pas limitée au temps du cours lui-même mais prend racine dans le passé de l'enseignant (voir Lahire, 2001), passé au cours duquel, par empilements de strates d'expériences, s'est construit comme un "idéaltype" d'enseignant, lequel apparaît en filigrane au cours des appréciations/ jugements/ maximes suscités par le déroulement du cours de langue visionné a posteriori. L'expérience passée est convoquée, pour la comparer par exemple à l'expérience présente. Les actions en classe ne sont pas seulement motivées par l'immédiateté des situations, par les réactions des étudiants ou 
par les obligations liées au programme, mais aussi par un certain nombre de principes méthodologiques qui préexistent, de "théories personnelles" qui se sont construites (par formation, expérience, observation...), ce qui donne à penser que l'action d'enseignement est basée sur des opinions ou des principes plus ou moins rationnels qui accompagnent ou sous-tendent l'interaction. Plus ou moins connus des protagonistes eux-mêmes.

Le point que nous allons aborder pour illustrer cet appel à un "extérieur de la classe" touche à l'identité langagière sociale des participantes au cours de Valérie (corpus Corny). Il se trouve que dans le cas de ce public de femmes travaillant en entreprise, et pour qui le cadre scolaire est assez lâche, l'enseignante fait plusieurs fois référence à leur rapport à la langue en-dehors de la classe.

\begin{abstract}
je ne sais pas si ça les intéresse c'est l'actualité + moi j'trouve ça important quand on habite dans un pays + euh si on veut + converser en français + de savoir c'qui s'y passe + euh on va dans un café on écoute les gens ils n'arrêtent pas d'parler de c'qu'ils entendent à la radio ++ elles pour certaines d'entre elles par exemple XXX ça fait faire 30 ans qu'elle est en France ++ euh les autres c'est plus récent + mais elle a pas du tout ni le réflexe du journal ni le réflexe de la radio juste la télé chinoise et TF1 (rires) non j'rigole mais j'leur ai expliqué un p'tit peu que TF1 c'était pas euh une chaîne comme les autres + et donc elles ont une grande culture du fait divers qui fait très peur (rires) si tu veux (rires) mais si non y'a pas vraiment de culture d'information quoi
\end{abstract} obstacle.
L'enseignante évoque les pratiques langagières des apprenantes en-dehors de la classe: elles ne lisent pas, elles n'écoutent pas la radio française, elles ne possèdent pas de culture d'information. La comparaison se fait entre ce public et un public "idéal" qui, pour l'enseignante, serait curieux et s'efforcerait de participer à la vie sociale du pays : " Moi j'trouve ça important quand on habite dans un pays + euh si on veut + converser en français + de savoir c'qui s'y passe + euh on va dans un café on écoute les gens".

Elle dresse comme un "portrait langagier" de ce public qui n'est pas habitué à être exposé à des discours en français, ce qui constitue un fait à prendre en compte et sans doute un

Tout au long de la verbalisation, ce portrait s'enrichit. Il comporte l'usage de la langue française dans la vie quotidienne, le rapport au dictionnaire, le goût pour les interactions de personne à personne, le désir de la traduction, du mot à mot, les difficultés à porter une attention trop longue à des discours écrits. Que les apprenantes aient envie de parler de leur quotidien, d'entamer une interaction de type plus intime, "ma fille, elle a un bouton" ${ }^{14}$ et redeviennent plus "femmes" qu'apprenantes met en danger le format de l'interaction didactique avec ses buts. Les désirs langagiers sont commentés par l'enseignante : est-ce "professionnel" de laisser ces thèmes entrer dans la classe ? Du point de vue du public, le désir exprimé c'est de parler du corps, de papoter, de parler de ce qui arrive dans leur vie, leur famille. Mais le danger que ressent l'enseignante, malgré son désir de répondre à cette attente c'est la déconstruction de la didacticité, la transformation de la classe en "salon de thé" qui apparaît comme une menace de destruction du genre professionnel qu'elle incarne.

Et parce que cette classe de langue a un contour institutionnel peu rigide, qu'elle pourrait devenir autre chose qu'une classe pour se transformer assez aisément en "salon de thé", mot qui revient plusieurs fois dans la bouche de l'enseignante, on voit alors s'exprimer le 
souci de soi comme professeur. Il vient contrecarrer l'envie de ces femmes d'avoir une activité de langage plus "ordinaire".

L'action en classe ne se limite pas à ce qui se passe en classe, les verbalisations donnent une indication sur les enjeux et sur la manière dont l'enseignante englobe le contexte, l'identité des sujets apprenants et leurs pratiques langagières.

\section{Conclusion}

Le rôle et la représentation du métier que se fait l'enseignant ne sont pas absents de la manière dont se construisent et se gèrent les genres conversationnels inhérents à la classe.

Pour approcher les "dessous de la fabrique de l'action", il est nécessaire de franchir plusieurs étapes :

1- D'abord celle de l'action qui se déroule en classe avec le public d'apprenants : selon des buts pédagogiques, selon le programme, selon les publics, en intégrant les contraintes temporelles.

2 - Puis celle de la découverte de fabrique de l'action qui se fait grâce à la pensée rétroactive des enseignants. L'enseignant qui se découvre agir - en action - construit un discours souvent légitimant pour justifier son action. Fabrique-t-il alors du sens, de la signification qui n'était pas présente au moment même? De quoi s'agit-il quand il se regarde agir par le biais de la rétroaction vidéo? Rend-il compte de son action ou bien fabrique-t-il une nouvelle image de ce qu'il a fait, avec le temps, la distance ? Toujours estil que c'est seulement par ce moyen que nous pouvons espérer tempérer la fugacité de l'action, cette action dont Arendt (1961/1983, voir le chapitre "L'action") dit qu'elle est à la fois infinie et toujours mouvante et glissante.

49 3- Enfin arrive l'étape d'analyse de l'action, qui concerne le chercheur se penchant sur les verbalisations d'enseignants. A partir des données qu'il a obtenues en filmant, enregistrant, et en s'entretenant avec les enseignants, il repère des constantes, des fractures, des dilemmes, il met en relation des termes qui reviennent, il opère des recoupements, il est à l'affût d'indices. Il est vrai qu'il risque de "fabriquer" une interprétation. On peut la penser incomplète mais, sans ce mouvement, cet arrêt sur images, l'action enseignante ne se donnerait pas à voir comme fabrique complexe.

Qu'en conclure quant au statut de la langue en classe ? Le souci de la langue n'est pas tant un souci de la langue en tant que telle, mais un souci de la langue apprise, à apprendre. La langue est un objet d'attente, voire de désir, aussi bien pour l'enseignant qui veut la transmettre que pour les apprenants qui tentent de se l'approprier. Elle apparait sous des formes très différentes et qui restent à étudier. Objet de la transmission, tout tourne indirectement autour d'elle. La classe produit des genres conversationnels qui lui sont propres : activité pédagogique, appel, bavardage, consignes, etc., mais les pratiques de la langue hors de la classe sont aussi présentes. 


\section{BIBLIOGRAPHIE}

Aguilar, J. \& Cicurel, F. (2014, dir.). "Pensée enseignante et didactique des langues". Recherches \& Applications $\mathrm{n}^{\circ} 56$. Paris : CLÉ International.

Arendt, H. (1961/1983, trad. fr.). Condition de l'homme moderne. Paris : Calmann-Lévy.

Arendt, H. (1972/1989, trad. fr). La crise de la culture. Huit exercices de pensée politique. Paris :

Gallimard, Collection Folio essais.

Bigot, V. (1996). "Converser en classe de langue : mythe ou réalité ?". Les Carnets du Cediscor 4.

Paris : Presses de la Sorbonne nouvelle. pp. 33-46.

Bigot, V. \& Cadet, L. (dir.). (2011). Discours d'enseignants sur leur action en classe. Enjeux théoriques et enjeux de formation. Paris : Riveneuve éditions.

Cicurel, F. (1985). Parole sur parole ou le métalangage en classe de langue. Paris : CLÉ international. Consultable sur le site :http://www.ilpga.univ-paris3.fr/pages-personnelles/francine-cicurel/ Parole-sur-parole.pdf.

Cicurel, F. (2011). "Le dire sur le faire : un retour (possible ?) sur l'action d'enseignement". In Bigot, V. \& Cadet, L. (dir.). Discours d'enseignants sur leur action en classe. Enjeux théoriques et enjeux de formation. Paris : Riveneuve éditions. pp. 51-69.

Cicurel, F. (2011). Les interactions dans l'enseignement des langues. Agir professoral et pratiques de classe. Paris : Didier.

Cicurel, F. \& Bigot, V. (dir.). (2005). Les interactions en classe de langue, Le français dans le monde : Recherches et applications. Paris : CLÉ international.

Clot, Y. et Faïta, D. (2000). "Genres et styles en analyse du travail. Concepts et méthodes". Travailler $\mathrm{n}^{\circ}$ 4. pp.7-42.

Clot, Y., Faïta, D., Fernandez, G., Scheller, L. (2001). "Entretiens en auto-confrontation croisée : une méthode en clinique de l'activité". Éducation permanente 146. pp.17-25.

Filliettaz, L. (2002). La parole en action. Éléments de pragmatique psycho-sociale. Québec : Éditions Nota bene.

Ginabat, H. (2006). La planification dans l'agir enseignant, Master de Recherche en Didactique du français et des langues. Paris : université Paris III-Sorbonne nouvelle.

Gumperz, J. (1989, trad. fr.). Engager la conversation. Introduction à la sociolinguistique interactionnelle. Paris : Les éditions de minuit.

Lahire, B. (2001). L'homme pluriel. Les ressorts de l'action. Paris : Hachette Littératures.

Muller, C. \& Delorme, V. (2014). "Ambivalence, adaptation et résistance : lorsque l'enseignant de langue est confronté à des réactions non planifiées d'apprenants". In Aguilar, J. \& Cicurel, F., (dir.). "Pensée enseignante et didactique des langues". Recherches \& Applications n 56. Paris : CLÉ international. pp. 33-63.

Perrenoud, Ph. (1996, 2e éd. 1999). Enseigner : agir dans l'urgence, décider dans l'incertitude. Savoirs et compétences dans un métier complexe. Paris : ESF. 
Plazaola Giger, I. \& Friedrich, J. (2005). "Comment l'agent met-il son action en mots ? Analyse d'entretiens auprès d'enseignants". In Filliettaz, L. \& Bronckart, J.-P. (dir.) L'analyse des actions et des discours en situation de travail. Concepts, méthodes et applications, Louvain-la-Neuve : Peeters. pp. 241-261.

Plazaola Giger, I. \& Stroumza, K. (dir.). (2007). Paroles de praticiens et description de l'activité. Problématisation méthodologique pour la formation et la recherche. Bruxelles : De Boeck.

Theureau, J. (2005). "Les méthodes de construction de données du programme de recherche sur les cours d'action et leur articulation collective, et la didactique des activités physiques et sportives". Impulsion 4. pp. 281-301.

Theureau, J. (2010). "Les entretiens d'autoconfrontation et de remise en situation par les traces matérielles et le programme de recherche 'cours d'action'". Revue d'Anthropologie des Connaissances, Vol. 4, $\mathrm{n}^{\circ} 2$ 2, 2010. pp. 287-322.

Schön, D. (1996). "À la recherche d'une nouvelle épistémologie de la pratique et de ce qu'elle implique pour l'éducation des adultes". In Barbier, J.-M. (dir.). Savoirs théoriques et savoirs d'action. Paris : PUF.

Schütz, A., trad. fr., (1998). Éléments de sociologie phénoménologique. Paris : L'Harmattan.

Sinclair, J. McH, Coulthard, R.M. (1975). Towards an Analysis of Discourse. Londres, Oxford: University Press.

Tochon, F. V. (1993). L'enseignant expert. Paris : Nathan pédagogie.

Von Cranach, M. et al. (1982). Goal-Directed Action. Londres : Academic Press.

Xue, L. (2014). "Quelques réflexions sur la fabrique de l'action enseignante dans une perspective diachronique". Dans ce recueil.

Zazzo, M. (2014). Reconfigurations de l'expérience d'enseignement à travers 3 entretiens d'autoconfrontation. Mémoire de Master Recherche, Université Sorbonne nouvelle Paris 3.

Woods, D. (1996). Teacher Cognition in Language Teaching. Beliefs, decision-making and classroom practice. Cambridge : Applied Linguistics.

\section{NOTES}

1. Voir les travaux du groupe Idap du centre de recherches Diltec, université Sorbonne nouvelle Paris 3, par exemple Bigot \& Cadet 2011 ou Aguilar \& Cicurel 2014.

2. Voir Theureau (2005) ; pour les techniques de l'auto-confrontation simple et croisée, Clot, Faïta, Fernandez, Scheller (2001) et pour ce qui est des enseignants, Woods (1996), Plazaola Giger \& Friedrich (2005); Cicurel (2011).

3. À propos de l'auto-confrontation: "Introduit dans sa forme actuelle par Von Cranach et sa "théorie de l'action dirigée vers un but" dans les années 80 (Theureau, 2005), l'entretien d'autoconfrontation (ou self-confrontation) est une méthode d'analyse de l'activité humaine consistant à confronter un ou plusieurs participants à une activité en les incitant à la commenter, en présence d'un interlocuteur. L'activité est généralement présentée sous forme d'enregistrement vidéo représentation la plus objective et complète de l'activité telle qu'elle a eu lieu -, mais peut selon les situations également comprendre des données audio, voire transcrites". Site consulté : http:// edutechwiki.unige.ch/fr/Entretien_d'autoconfrontation. Voir aussi dans le numéro Recherches \& applications $\mathrm{n}^{\circ} 56$ le guide méthodologique pour l'entretien EAC rédigé par le groupe Idap. 
4. "Selon l'hypothèse de la conscience préréflexive : un acteur humain peut à chaque instant, moyennant la réunion des conditions favorables, montrer, mimer, simuler, raconter et commenter son activité - ses éléments comme son organisation temporelle complexe - à un observateur interlocuteur" (Theureau, $2010: 3)$.

5. Le dictionnaire Le Robert donne comme définition de verbaliser: "Exprimer, extérioriser quelque chose au moyen du langage".

6. Il agit devant un public qui attend quelque chose de lui, il a un programme à mettre en place, il doit maîtriser des méthodologies ou des manières de faire un cours et, en tout cas, adapter constamment ce qu'il dit à un public qui peut souhaiter que le rythme s'accélère, qui risque à tout moment de se désintéresser du cours.

7. Voir Arendt, H. (1961/1983, trad. fr.). Condition de l'homme moderne et Arendt H. (1972/1989), La crise de la culture.

8. Les séquences de verbalisations sont extraites des corpus constitués par les chercheurs de l'Idap - Diltec et les étudiants en Master 2 Didactique du français et des langues, université Sorbonne Nouvelle-Paris 3. Dans la présente étude le corpus Corny met en présence une jeune enseignante, Valérie, donnant des cours de FLE à des travailleuses habitant en France, le corpus Ginabat une enseignante chevronnée s'adressant à des étudiants chinois suivant des cours de FLE en milieu universitaire. Le corpus Gaya est également une situation d'enseignement du français à des publics chinois dans une école de commerce.

9. L'enseignant est celui qui donne la parole, apprécie les paroles des apprenants, est en charge de la conduite des interactions, gère et surveille le déroulement des activités, etc. Il est aussi celui qui est responsable de l'interaction et de sa bonne marche.

10. Le corpus Corny rassemble des données d'interactions de cours de FLE s'adressant à des femmes travailleuses, résidant en France depuis parfois quelques années. Elles ont tendance à demeurer dans leur communauté d'origine (cambodgienne, sri-lankaise etc.). Le filmage d'une classe a donné lieu à une auto-confrontation dont sont tirés la plupart des extraits ici donnés.

Code de conventions de transcription : pause + ; intonation montante $\uparrow ;$ Majuscule $=$ accentuation. Sont soulignés en gras les termes renvoyant à l'acte de parler : discuter, s'exprimer, reformuler, etc.

11. Voir la comparaison que propose Zazzo (2014) de trois enseignants opérant dans un même contexte tout en ayant une formation comparable et se caractérisant cependant par un style professoral différent.

12. Valérie déclare par ailleurs dans l'entretien sa forte envie d'écouter ses apprenantes (des femmes asiatiques résidant en France et travaillant en entreprise comme caissières).

13. Roy Jacobsen, 2014, Le prodige, trad. du norvégien par Alain Gnaedig, Gallimard.

14. C'est ce que Valérie rapporte à un autre moment de la verbalisation lorsqu'elle imagine les thèmes conversationnels que ses apprenantes aimeraient aborder.

\section{RÉSUMÉS}

Une interaction de type didactique dans le cadre de l'enseignement d'une langue étrangère a ses règles et ses codes de communication. Émergent des genres conversationnels caractérisés par des normes interactionnelles contraintes par le genre didactique d'une situation donnée. Cet article 
se penche sur la manière dont s'agence et "se fabrique de la parole" en classe de langue en s'appuyant sur quelques exemples extraits d'auto-confrontation. À la lumière d'un corpus constitué par des verbalisations d'enseignants de cours de FLE, on s'attache à y détecter la manière d'évoquer les activités langagières que le cours est supposé susciter. Cette investigation devrait permettre de mettre en valeur l'apport de données de type verbalisations pour la didactique des langues.

Within the context of foreign language teaching, didactic interactions have their own rules and codes of communication. The conversational styles that emerge are characterized by interactional norms as they are defined by the didactic situation at hand. Based on several examples taken from self-confrontation, this article looks at the ways in which speech organizes and creates itself within the language classroom. In light of a body of research made up of the verbalizations of teachers of French as a foreign language, we've endeavored to detect the ways to evoke language activities that the class supposedly incites. This investigation should serve to valorize the contribution to the field of applied linguistics of data in the form of verbalizations confronted with the act of teaching.

\section{INDEX}

Keywords : action, didactic interaction, interactive norms, interactive style, self-confrontation, teacher talk, verbalization

Mots-clés : action, auto-confrontation, genre conversationnel, interaction didactique, norme interactionnelle, parole enseignante, verbalisation

\section{AUTEUR}

\section{FRANCINE CICUREL}

Université Sorbonne Nouvelle-Paris 3, Diltec - Idap, France

Francine Cicurel est professeure émérite à l'université Sorbonne nouvelle - Paris 3, laboratoire Diltec (groupe Idap).

Toile : http://www.univ-paris3.fr/idap-interactions-didactiques-et-agir-professoral-119992.kjsp

Courriel : francine.cicurel[at]univ-paris3.fr. 